\title{
Influencia del uso del aula virtual en los procesos pedagógicos de los estudiantes de Zegel Ipae
}

\author{
Influence of the use of the virtual classroom in the pedagogical \\ processes of ZegelIpae students
}

Influência do uso da sala de aula virtual nos processos

pedagógicos dos alunos da ZegelIpae

ROXANA HAYDEÉ OLIVERA ROQUE ${ }^{1}$

\begin{abstract}
RESUMEN
Con el fin de determinar la influencia que tiene el uso del aula virtual en los procesos pedagógicos de los estudiantes, se aplicó un diseño cuasi experimental con una muestra de 64 estudiantes, la cual fue dividida en dos grupos 32 para el grupo de control y 32 para el grupo experimental (los que usaron aula virtual) se aplicó un post test (cuestionario) donde se evaluó los cuatro primeros procesos pedagógicos siguientes: la problematización, propósito-organización, motivación-saberes previos y gestión-acompañamiento, utilizando la "U” de Mann-Whitney para los resultados. Para evaluar la quinta dimensión: evaluación, se aplicó una pre y post prueba de conocimientos, utilizando la T de Student. Llegando a la conclusión que el uso de las aulas virtuales mejoró significativamente los procesos pedagógicos en los estudiantes del grupo experimental ya que estos lograron resultados superiores en comparación a los del grupo de control que trabajaron con la metodología tradicional.
\end{abstract}

Palabras clave: Aula virtual; procesos pedagógicos; motivación; evaluación; aprendizaje.

\begin{abstract}
In order to determine the influence that the use of the virtual classroom has on the pedagogical processes of the students, a quasi-experimental design was applied with a sample of 64 students; which was divided into two groups: 32 for the control group and 32 for the experimental group (those who used a virtual classroom). A posttest (questionnaire) was applied in which the following four pedagogical processes were evaluated: problematization, purpose-organization, motivation-previous knowledge and management-accompaniment; using the Mann-Whitney " $U$ " for results. In order to evaluate the fifth dimension: evaluation; a pre and post knowledge test was applied, using the Student's T test. Reaching the conclusion that the use of virtual classrooms significantly improved the pedagogical processes in the students of the experimental group since they achieved superior results compared to those of the control group that worked with the traditional methodology.
\end{abstract}


Keywords: Virtual classroom; pedagogical processes; motivation; evaluation; learning.

\section{RESUMO}

Para determinar a influência que o uso da sala de aula virtual exerce sobre os processos pedagógicos dos alunos, foi aplicado um desenho quase experimental com uma amostra de 64 alunos; o qual foi dividido em dois grupos: 32 para o grupo controle e 32 para o grupo experimental (aqueles que usaram uma sala de aula virtual). Foi aplicado um pós-teste (questionário), no qual foram avaliados os quatro processos pedagógicos a seguir: problematização, organização de propósitos, conhecimento prévio de motivação e acompanhamento gerencial; usando o Mann-Whitney "U” para obter resultados. Avaliar a quinta dimensão: avaliação; Foi aplicado um teste pré e pós conhecimento, utilizando o teste T de Student. Chegando à conclusão de que o uso de salas de aula virtuais melhorou significativamente os processos pedagógicos nos alunos do grupo experimental, pois alcançaram resultados superiores aos do grupo controle que trabalhou com a metodologia tradicional.

Palavras-chave: Aula virtual; processos pedagógicos; motivação; avaliação; aprendendo.

\section{INTRODUCCIÓN}

Vemos en estos días en casi todas las sociedades, personas desempeñando ocupaciones diferentes y asumiendo responsabilidades en las actividades del hogar, más aún; en el caso de la mujer, conciliar su papel de ama de casa con su rol de agente económico productivo y que, por razones laborales, familiares, de edad, lugar de residencia, entre otros; no pueden asistir físicamente a un instituto o universidad y dejan de perseguir ese crecimiento profesional.

Esa es una de las razones por lo que, en la actualidad, instituciones educativas enfocan sus enseñanzas en la modalidad virtual, en las TIC -en el campo de la docencia están tomando fuerza a nivel mundial debido a su aporte- ya que el educando de hoy tiene un acceso más fácil y rápido a la información, a la tecnología, con experiencias de aprendizajes innovadoras. Por estas razones existen muchas instituciones que cuentan con plataformas virtuales que ofrecen servicios de aprendizaje para que las personas puedan continuar su vida profesional sin descuidar su vida personal, con horarios flexibles, con accesibilidad a los contenidos, etc.

Pero la inquietud que se da en la actualidad de quien anhela estudiar, radica en la duda que genera las clases presenciales versus las virtuales, por tanto, este potencial estudiante desconoce si le ira bien, si tendrá algún problema en la comprensión o si los materiales brindados serán suficientes para comprender el tema nuevo. Entonces aquí viene la preocupación del gestor educativo responsable de la institución, verificar si realmente se está logrando la satisfacción de sus clientes (estudiantes). Para eso el papel del aula virtual debe garantizar el buen cumplimiento de los procesos pedagógicos.

En el aula virtual los docentes pueden resaltar el aprendizaje de manera reflexiva, usando la crítica y sobre todo la creatividad, aprendiendo a través de la indagación de muchas fuentes 
y estrategias de información puestas como contenido, desarrollando así en el proceso competencias y capacidades para luego evaluarlas (Minedu, 2014).

La Web del maestro CMF (2015) define los procesos pedagógicos como las actividades que se puede desarrollar en cualquier momento, esto diferencia a los procesos de los momentos; que el docente realiza para que pueda dosificar el aprendizaje del estudiante con la intención de garantizar la construcción de conocimientos y desarrollar competencias consideran entonces como procesos pedagógicos. Por ello investigaremos: ¿Cuál es la influencia del uso del aula virtual en los procesos pedagógicos de los estudiantes de la carrera de administración del Instituto Superior Zegel Ipae turno noche sede San Juan de Lurigancho 2018-I?

Los Procesos Pedagógicos según (Las Rutas del Aprendizaje, 2017) son actividades que se desarrollan de manera intencional y sirven para conducir el aprendizaje significativo y así poder construir los conocimientos, desarrollando competencias para la vida cotidiana, hay que resaltar que estos procesos son permanentes y se pueden dar o recordar en cualquier momento se crea conveniente. Estos procesos pedagógicos son:

- Problematización: se trata de presentarle al estudiante casos cotidianos donde el desafío o reto de solucionar ese problema esté presente que serán de interés, o de necesidad, o 1 expectativa del tema la cual se va a trabajar.

- Propósito y organización: lo que se da a conocer en este proceso es el logro de aprendizaje, es decir el propósito que tendrá de estudiar ese tema para su vida profesional o cotidiana y no solo eso también con que tema lo hará y como será evaluado.

- Motivación: nos dice que para garantizar el éxito del desarrollo de aprendizaje los estudiantes tienen que estar motivados, eso hará que ellos resuelvan los problemas por voluntad y gran expectativa hasta el final de los procesos

- Gestión y acompañamiento: En este proceso se realiza todo lo que conoce el docente en cuestión de manejo y preparación para el uso adecuado de estrategias y secuencias didácticas; como también acompañar a los estudiantes en los momentos de ejecución aplicando la reflexión, la capacidad de análisis, fomentando el dialogo, e impulsando la crítica, etc., para garantizar el buen desenvolvimiento de los estudiantes en la construcción de sus conocimientos.

- Evaluación: en este proceso se da de principio a fin donde el docente al final de la sesión tiene que ver si se logró lo que se esperaba y que con evidencias cuenta para demostrar el desempeño esperado la cual aplicaran las competencias desarrolladas durante los procesos.

El objetivo general de esta investigación fue determinar cómo el uso del aula virtual influye en los procesos pedagógicos de los estudiantes de administración del Instituto Superior Zegel Ipae turno noche- sede San Juan de Lurigancho 2018-I. Mientras que los objetivos específicos fueron, determinar la influencia del uso del aula virtual en el proceso problematización de los estudiantes; determinar la influencia del uso del aula virtual en el proceso propósito y organización de los estudiantes; determinar la influencia del uso del aula virtual en el proceso motivación y saberes previos de los estudiantes; determinar la influencia del 
uso del aula virtual en el proceso gestión y acompañamiento de los estudiantes; y determinar la influencia del uso del aula virtual en el proceso evaluación de los estudiantes de la carrera de administración del Instituto objeto de estudio.

Aguilar (2014) en su tesis se propuso "evaluar la influencia de las aulas virtuales en el aprendizaje por competencias de los estudiantes del curso de Internado Estomatológico de la Facultad de Odontología de la Universidad de San Martín de Porres en el año 2013” (p. 5), en una investigación explicativa de diseño transversal, utilizando la técnica de encuesta $\mathrm{y}$, como instrumento test de evaluación que fue aplicado a 260 estudiantes divididos en 130 para el grupo experimental y 130 para el grupo de control, llegando a la conclusión que "los resultados obtenidos al finalizar esta investigación nos prueban que las aulas virtuales influyen significativamente en el aprendizaje por competencias de los estudiantes del curso de Internado Estomatológico de la Facultad de Odontología de la Universidad de San Martín de Porres" (p. 66).

Rodrigo (2015) en su tesis se propuso "demostrar que la educación virtual es una alternativa didáctica para el desarrollo de competencias genéricas de Alfa Tuning Latinoamérica y para la adopción de buenas prácticas educativas dentro del proceso de enseñanza-aprendizaje" (p. 15), en una investigación descriptiva, de diseño no experimental, utilizando la técnica de encuesta que fue aplicado a 11 docentes de maestría y en la segunda etapa a 10 alumnos, llegando a la conclusión que "la Educación Virtual Universitaria es una alternativa de calidad de enseñanza y aprendizaje frente a la Educación Presencial [...] permite la actualización permanente de las actividades de aprendizaje a través de las plataformas virtuales educativas" (p. 229).

García (2015) en su tesis propuso "analizar el uso que hacen los docentes del programa de ingeniería de sonido, de las aulas virtuales como apoyo a las clases presenciales en la Universidad de San Buenaventura, Bogotá" (p. 21), en una investigación descriptiva, de diseño no experimental, de corte transversal, utilizando la técnica de encuesta y que fue aplicado a los "docentes del programa de Ingeniería de sonido" (p. 43), llegando a la conclusión "que el uso de las aulas virtuales por parte de los docentes del programa Ingeniería de Sonido, de la Universidad de San Buenaventura, sede Bogotá, es poco. El uso limitado de recursos y actividades, así como las opiniones dadas por los docentes, evidencian un bajo nivel de apropiación tecnológica y pedagógica en la aplicación de las aulas virtuales como herramientas de apoyo en el modelo presencial lo cual limita el potencial de este tipo de recursos en la labor de enseñanza y aprendizaje" (p. 77).

Cepeda (2017) en su tesis se propuso "determinar como la estrategia metodológica del uso de aulas virtuales influye en el proceso de enseñanza aprendizaje de la asignatura de Psicología Educativa I en los estudiantes de segundo semestre de la carrera de Psicología Educativa de la Facultad de Ciencias de la Educación, Humanas y Tecnologías de la UN$\mathrm{ACH}$ en el período académico 2015" (p. 6), en una investigación explicativa, de diseño cuasiexperimental, de corte longitudinal, utilizando la técnica de la encuesta y, como instrumento el cuestionario que fue aplicado a 31 del grupo experimental y 31 al grupo de control, llegando a la conclusión que "que las aulas virtuales influyen significativamente en el aprendizaje por competencias de los estudiantes del curso de Internado Estomatológico de la Facultad de Odontología de la Universidad de San Martín de Porres” (p. 66). 


\section{METODOLOGÍA}

La población objetivo corresponde a los estudiantes del primer ciclo, matriculados en la asignatura de estadística de la carrera de Administración de Empresas escuela de gestión y está conformado por un total de 150 estudiantes matriculados en el semestre 2018-I. Los grupos de la muestra han sido elegidos al azar, de forma que cada grupo de la población tuvo igual oportunidad de salir en la muestra a través de un muestreo no probabilístico a criterio del autor. Teniendo en consideración dos grupos: cuasi experimental: Estuvo conformado por 64 estudiantes (32 para el grupo de control y 32 para el grupo experimental) de la carrera de administración del Instituto Superior Zegel Ipae turno noche- sede San Juan de Lurigancho.

Para el recojo de información se utilizó como técnica la encuesta, estas se realizaron en una sesión de clase cada sesión de clase la cual duró 2 horas pedagógicas de 45 minutos, de esta manera, paralelamente se pudo observar cómo los estudiantes interactuaron con el aula virtual y como fue la interacción sin hacer uso de este. A final del experimento se aplicó el instrumento del cuestionario (post test) tipo escala Likert, los cuales están compuestos por un conjunto de preguntas con respecto a las variables que están sujetas a medición, y que son elaborados teniendo en cuenta los objetivos de la investigación, donde se evaluó los procesos pedagógicos siguientes: la problematización, propósito-organización, motivación-saberes previos y gestión-acompañamiento. La información recabada fue procesada con técnicas estadísticas: la prueba "U" de Mann-Whitney para las 4 primeras dimensiones. Para evaluar la quinta dimensión (evaluación) se aplicó una pre y post prueba de conocimientos, en los resultados obtenidos se aplicó la prueba de T de Student para el grupo experimental y control.

Para el procesamiento y análisis de datos se formaron dos grupos: el del control y el experimental. El grupo de control formado por los 32 estudiantes del paralelo A, que trabajaron de manera tradicional, y el grupo experimental formado por los 32 estudiantes del paralelo $\mathrm{B}$, que trabajaron con el apoyo del uso del aula virtual, dando un total de 64 participantes en la investigación. Al final del curso se implementó (no para fines de medición) una nivelación para el grupo de control, para que no se vean afectados los integrantes del grupo, en esa nivelación se utilizó el aula virtual.

Para la elaboración de la sesión de clase, se utilizó el aula virtual, instalada en los servidores del instituto superior Zegel Ipae, la cual tienen acceso todos los docentes y estudiantes. Para el análisis de datos se empleó el programa estadístico SPSS 24, con el cual se realizaron los siguientes análisis:

1. Medidas de tendencia central y de variabilidad para el análisis cuantitativo de las variables de estudio.

2. Prueba de Kolmogórov-Smirnov para determinar si los datos se aproximan a una distribución normal y elegir el tipo de estadística adecuada (paramétrica o no paramétrica). 
3. Prueba "U" de Mann-Whitney para las cuatro primeras dimensiones y realizar las comparaciones entre el grupo experimental y el control en el post test de las respuestas aplicadas en el cuestionario.

4. Prueba $\mathrm{T}$ de Student para la última dimensión y realizar las comparaciones de las calificaciones entre el grupo experimental y el control tomadas en el pre y la posprueba.

5. Finalmente, se presentaron los resultados, conclusiones del trabajo de investigación.

\section{RESULTADOS}

\section{Para la dimensión problematización}

Tabla 1

Media y desviación típica de los grupos en la dimensión problematización

\begin{tabular}{ccccccc} 
& \multicolumn{3}{c}{ grupo experimental } & \multicolumn{3}{c}{ grupo control } \\
\cline { 2 - 7 } & Media & $\begin{array}{c}\text { Desviación } \\
\text { típica }\end{array}$ & Recuento & Media & $\begin{array}{c}\text { Desviación } \\
\text { típica }\end{array}$ & Recuento \\
Problematización & 17.938 & 0.948 & 32 & 7.875 & 1.963 & 32 \\
\hline
\end{tabular}

Fuente: Resultados obtenidos en software SPSS 24

Para el proceso pedagógico de la problematización, se observa una diferencia significativa en los puntajes al analizar la dimensión problematización de los estudiantes de la carrera de administración del Instituto Zegel Ipae (post test) de entre los estudiantes del grupo de control y experimental, siendo éstos últimos los que obtuvieron mayores puntajes lo siguiente: Los estudiantes del grupo experimental obtuvieron mejores resultados al analizar la dimensión problematización sus puntajes fueron $(17.938 \pm 0.948)$ después de la aplicación del aula virtual, respecto a los estudiantes del grupo de control que no utilizaron el aula virtual $(7.875 \pm 1.96)$ ver Tabla 1.

\section{Tabla 2}

Estadísticos de Mann-Whitney del proceso problematización

\begin{tabular}{lc}
\hline & Problematización \\
\hline U de Mann-Whitney & 0.000 \\
W de Wilcoxon & 528.000 \\
Z & -6.948 \\
Sig. asintót. (bilateral) & .000 \\
\hline
\end{tabular}

Fuente: Resultados obtenidos en software SPSS 24

Se observa de acuerdo a la prueba no paramétrica U de Mann-Whitney para muestras independientes, tanto para el grupo de control y experimental lo siguiente: Existe una diferencia estadísticamente significativa $\mathrm{P}=0.000$ (ver Tabla 2). 


\section{Para la dimensión propósito y organización}

Tabla 3

Media y desviación típica de los grupos en la dimensión propósito y organización

\begin{tabular}{|c|c|c|c|c|c|c|}
\hline & \multicolumn{6}{|c|}{ GRUPO } \\
\hline & \multicolumn{3}{|c|}{ grupo experimental } & \multicolumn{3}{|c|}{ grupo control } \\
\hline & Media & $\begin{array}{l}\text { Desviación } \\
\text { típica }\end{array}$ & Recuento & Media & $\begin{array}{l}\text { Desviación } \\
\text { típica }\end{array}$ & Recuento \\
\hline $\begin{array}{l}\text { Propósito y } \\
\text { organización }\end{array}$ & 13.563 & 0.982 & 32 & 5.531 & 1.218 & 32 \\
\hline
\end{tabular}

Fuente: Resultados obtenidos en software SPSS 24

Para el proceso pedagógico propósito y organización, se observa una diferencia significativa en los puntajes de los estudiantes de la carrera de administración del Instituto Zegel Ipae (post test) de entre los estudiantes del grupo de control y experimental, siendo éstos últimos los que obtuvieron mayores puntajes lo siguiente: Los estudiantes del grupo experimental obtuvieron mejores resultados al analizar la dimensión propósito y organización sus puntajes fueron (13.563 \pm 0.982$)$ después de la aplicación del aula virtual, respecto a los estudiantes del grupo de control que no utilizaron el aula virtual $(5.531 \pm 1.218)$ ver Tabla 3.

Tabla 4

Estadísticos de Mann-Whitney del proceso propósito y organización

\begin{tabular}{lc}
\hline & Propósito y organización \\
\hline U de Mann-Whitney & 0.000 \\
W de Wilcoxon & 528.000 \\
Z & -6.963 \\
Sig. asintót. (bilateral) & .000 \\
\hline
\end{tabular}

Fuente: Resultados obtenidos en software SPSS 24

Se observa de acuerdo a la prueba no paramétrica U de Mann-Whitney para muestras independientes, tanto para el grupo de control y experimental lo siguiente: Existe una diferencia estadísticamente significativa $\mathrm{P}=0.000$ (ver Tabla 4). 


\section{Para la dimensión motivación y saberes previos}

Tabla 5

Media y desviación típica de los grupos en la dimensión motivación y saberes previos

\begin{tabular}{ccccccc} 
& \multicolumn{3}{c}{ grupo experimental } & \multicolumn{3}{c}{ grupo control } \\
\cline { 2 - 7 } & Media & $\begin{array}{c}\text { Desviación } \\
\text { típica }\end{array}$ & Recuento & Media & $\begin{array}{c}\text { Desviación } \\
\text { típica }\end{array}$ & Recuento \\
$\begin{array}{c}\text { Motivación y } \\
\text { saberes previos }\end{array}$ & 18.094 & 1.027 & 32 & 7.625 & 1.792 & 32 \\
\hline
\end{tabular}

Fuente: Resultados obtenidos en software SPSS 24

Para el proceso pedagógico motivación y saberes previos, se observa una diferencia significativa en los puntajes de los estudiantes de la carrera de administración del Instituto Zegel Ipae (post test) de entre los estudiantes del grupo de control y experimental, siendo éstos últimos los que obtuvieron mayores puntajes. Los estudiantes del grupo experimental obtuvieron mejores resultados al analizar la dimensión motivación y saberes previos puntajes fueron $(18.09 \pm 1.02)$ después de la aplicación del aula virtual, respecto a los estudiantes del grupo de control que no utilizaron el aula virtual $(7.625 \pm 1.792)$ ver Tabla 5.

Tabla 6

Estadísticos de Mann-Whitney del proceso motivación y saberes previos

\begin{tabular}{lc}
\hline & Motivación y saberes previos \\
\hline U de Mann-Whitney & 0.000 \\
W de Wilcoxon & 528.000 \\
Z & -6.922 \\
Sig. asintót. (bilateral) & .000 \\
\hline
\end{tabular}

Fuente: Resultados obtenidos en software SPSS 24

Se observa de acuerdo a la prueba no paramétrica U de Mann-Whitney para muestras independientes, tanto para el grupo de control y experimental lo siguiente: Existe una diferencia estadísticamente significativa $\mathrm{P}=0.000$ (ver Tabla 6).

\section{Para la dimensión gestión y acompañamiento}

\section{Tabla 7}

Media y desviación típica de los grupos en la dimensión gestión y acompañamiento

\begin{tabular}{ccccccc} 
& \multicolumn{3}{c}{ Grupo experimental } & \multicolumn{3}{c}{ Grupo control } \\
\cline { 2 - 7 } & Media & Desviación típica & Recuento & Media & Desviación típica & Recuento \\
Gestión & 22.688 & 0.896 & 32 & 9.875 & 1.431 & 32 \\
\hline
\end{tabular}

Fuente: Resultados obtenidos en software SPSS 24 
Para el proceso pedagógico gestión y acompañamiento, se observa una diferencia significativa en los puntajes de los estudiantes de la carrera de administración del Instituto Zegel Ipae (post test) de entre los estudiantes del grupo de control y experimental, siendo éstos últimos los que obtuvieron mayores puntajes lo siguiente: Los estudiantes del grupo experimental obtuvieron mejores resultados al analizar la dimensión gestión y acompañamiento puntajes fueron $(22.688 \pm 0.896)$ después de la aplicación del aula virtual, respecto a los estudiantes del grupo de control que no utilizaron el aula virtual $(9.875 \pm 1.431)$ ver Tabla 7.

Tabla 8

Estadísticos de Mann-Whitney del proceso gestión y acompañamiento

\begin{tabular}{cc}
\hline & Gestión y acompañamiento \\
\hline U de Mann-Whitney & 0.000 \\
W de Wilcoxon & 528.000 \\
Z & -6.946 \\
Sig. asintót. (bilateral) & .000 \\
\hline
\end{tabular}

Fuente: Resultados obtenidos en software SPSS 24

Se observa de acuerdo a la prueba no paramétrica U de Mann-Whitney para muestras independientes, tanto para el grupo de control y experimental lo siguiente: Existe una diferencia estadísticamente significativa $\mathrm{P}=0.000$ (ver Tabla 8 ).

\section{Aplicación de estadístico a las pruebas en la dimensión evaluación}

\section{- En la pre evaluación}

Tabla 9

Media y desviación típica de los grupos en la pre prueba

\begin{tabular}{llllll}
\hline Grupo & & $\mathrm{N}$ & Media & Desviación típ. & $\begin{array}{c}\text { Error típ. de } \\
\text { la media }\end{array}$ \\
\hline \multirow{2}{*}{ Preprueba } & grupo experimental & 32 & 2.50 & 1.524 & .269 \\
& grupo control & 32 & 2.13 & 1.601 & .283 \\
\hline
\end{tabular}

Fuente: Resultados obtenidos en software SPSS 24

Con respecto al puntaje de la dimensión evaluación de los estudiantes de la carrera de administración del Instituto Zegel Ipae tanto para el grupo de control y experimental, se observó que los estudiantes del grupo experimental obtuvieron casi los mismos resultados $(2.5 \pm 1.5)$ antes del uso del aula virtual, respecto a los estudiantes del grupo de control $(2.13 \pm 1.6)$ ver Tabla 9. 
Tabla 10

Prueba T de Student para muestras independientes en el pre prueba

\begin{tabular}{|c|c|c|c|c|c|c|c|c|}
\hline & \multicolumn{7}{|c|}{ Prueba T para la igualdad de medias } \\
\hline & & \multirow[t]{2}{*}{$\mathrm{t}$} & \multirow[t]{2}{*}{ gl } & \multirow{2}{*}{$\begin{array}{l}\text { Sig. (bi- } \\
\text { lateral) }\end{array}$} & \multirow{2}{*}{$\begin{array}{l}\text { Diferen- } \\
\text { cia de } \\
\text { medias }\end{array}$} & \multirow{2}{*}{$\begin{array}{l}\text { Error } \\
\text { típ. de la } \\
\text { diferen- } \\
\text { cia }\end{array}$} & \multicolumn{2}{|c|}{$\begin{array}{c}95 \% \text { Intervalo de } \\
\text { confianza para la } \\
\text { diferencia }\end{array}$} \\
\hline & & & & & & & Inferior & Superior \\
\hline & $\begin{array}{c}\text { Se han } \\
\text { asumido } \\
\text { varianzas } \\
\text { iguales }\end{array}$ & .960 & 62 & .341 & .375 & .391 & -.406 & 1.156 \\
\hline Preprueba & $\begin{array}{c}\text { No se } \\
\text { han asu- } \\
\text { mido } \\
\text { varianzas } \\
\text { iguales }\end{array}$ & .960 & 61.848 & .341 & .375 & .391 & -.406 & 1.156 \\
\hline
\end{tabular}

Fuente: Resultados obtenidos en software SPSS 24

Si comparamos los resultados del grupo experimental con el de grupo de control en la pre prueba, tenemos que el nivel de significación de acuerdo a la prueba T Student para muestras independientes es 0.341 mostraron homogeneidad en los grupos ( $p>0.05)$. No existe una diferencia estadísticamente significativa, ver Tabla 10.

\section{- En la post evaluación}

Tabla 11

Media y desviación típica de los grupos en la post prueba

\begin{tabular}{llllll}
\hline Grupo de experimental & $\mathrm{N}$ & Media & Desviación típ. & $\begin{array}{l}\text { Error típ. de la } \\
\text { media }\end{array}$ \\
\hline \multirow{2}{*}{ Post prueba } & grupo experimental & 32 & 18.13 & 2.028 & .359 \\
& grupo control & 32 & 11.50 & 1.901 & .336 \\
\hline
\end{tabular}

Fuente: Resultados obtenidos en software SPSS 24

El puntaje de la dimensión evaluación de los estudiantes de los estudiantes de la carrera de administración del Instituto Zegel Ipae de acuerdo a la prueba paramétrica T Student para muestras independientes, tanto para el grupo de control y experimental según el post test. Los estudiantes del grupo experimental obtuvieron mejores resultados en sus puntajes de la dimensión evaluación $(18.13 \pm 2.02)$, después del uso del aula virtual, respecto a los estudiantes del grupo de control $(11.5 \pm 1.90)$ ver Tabla 11. 
Tabla 12

Prueba T de Student para muestras independientes en el post prueba

\begin{tabular}{|c|c|c|c|c|c|c|c|c|}
\hline & & Prueba & para la & ualdad de m & dias & & & \\
\hline & & & & $\begin{array}{l}\text { Sig. (bila- } \\
\text { teral) }\end{array}$ & $\begin{array}{l}\text { Diferencia } \\
\text { de medias }\end{array}$ & $\begin{array}{l}\text { Error típ. } \\
\text { de la dife- }\end{array}$ & $\begin{array}{l}95 \% \text { In } \\
\text { confianz } \\
\text { ferencia }\end{array}$ & $\begin{array}{l}\text { ervalo de } \\
\text { para la di- }\end{array}$ \\
\hline & & & & & & & Inferior & Superior \\
\hline$P \circ s t$ & $\begin{array}{l}\text { Se han } \\
\text { asumido } \\
\text { varianzas } \\
\text { iguales }\end{array}$ & 13.483 & 62 & .000 & 6.625 & .491 & 5.643 & 7.607 \\
\hline prueba & $\begin{array}{l}\text { No se han } \\
\text { asumido } \\
\text { varianzas } \\
\text { iguales }\end{array}$ & 13.483 & 61.741 & .000 & 6.625 & .491 & 5.643 & 7.607 \\
\hline
\end{tabular}

Fuente: Resultados obtenidos en software SPSS 24

Si comparamos los resultados del grupo experimental con el de grupo de control en la post prueba, tenemos que el nivel de significación de acuerdo a la prueba T Student para muestras independientes es 0.000 mostrando heterogeneidad en los grupos $(\mathrm{p}<0.05)$. Existe una diferencia estadísticamente significativa (ver Tabla 12).

\section{DISCUSIÓN}

Los resultados en cuanto a las hipótesis formuladas, nos muestran que:

Sobre la hipótesis general, de la aplicación no paramétrica de la U de Mann-Whitney se muestra que el margen de significancia es del 0.000 lo cual rechaza la hipótesis nula y acepta la hipótesis alternativa estableciendo que existe una influencia entre ellas por esto se concluye que "El uso del aula virtual influye significativamente en los procesos pedagógicos de los estudiantes de la carrera de administración del Instituto Zegel Ipae 2018-I.

En cuanto a la primera de las hipótesis específicas, de la aplicación de la prueba no paramétrica U de Mann-Whitney nos da un valor de significancia del 0.000 con lo cual validamos la hipótesis alterna que sugiere que "El uso del aula virtual influye significativamente en la dimensión problematización de los procesos pedagógicos de los estudiantes de la carrera de administración del Instituto Zegel Ipae; 2018-I.

En cuanto a la segunda de las hipótesis específicas, de la aplicación de la prueba no paramétrica U de Mann-Whitney nos da un valor de significancia del 0.000 con lo cual validamos la hipótesis alterna que sugiere que "El uso del aula virtual influye significativamente en la dimensión propósito y organización de los procesos pedagógicos de los estudiantes de la carrera de administración del Instituto Zegel Ipae; 2018-I.

En cuanto a la tercera de las hipótesis específicas, de la aplicación de la prueba no paramétrica U de Mann-Whitney nos da un valor de significancia del 0.000 con lo cual validamos la hipótesis alterna que sugiere que "El uso del aula virtual influye significativamente en la 
dimensión motivación y saberes previos de los procesos pedagógicos de los estudiantes de la carrera de administración del Instituto Zegel Ipae; 2018-I.

En cuanto a la cuarta de las hipótesis específicas, de la aplicación de la prueba no paramétrica U de Mann-Whitney nos da un valor de significancia del 0.000 con lo cual validamos la hipótesis alterna que sugiere que "El uso del aula virtual influye significativamente en la dimensión gestión y acompañamiento de los procesos pedagógicos de los estudiantes de la carrera de administración del Instituto Zegel Ipae; 2018-I.

En cuanto a la quinta de las hipótesis específicas, de la aplicación de la prueba no paramétrica U de Mann-Whitney nos da un valor de significancia del 0.000 con lo cual validamos la hipótesis alterna que sugiere que "El uso del aula virtual influye significativamente en la dimensión evaluación de los procesos pedagógicos de los estudiantes de la carrera de administración del Instituto Zegel Ipae; 2018-I.

\section{CONCLUSIONES}

Una mayor cantidad de estudiantes que utilizo el aula virtual tuvo más situaciones retadoras y desafiantes la cual incremento el interés de la clase a tratar a comparación de los que non usaron aula virtual. Los estudiantes que usaron el aula virtual mencionaron que tuvieron conocimiento del logro del aprendizaje que se esperaba en la sesión de clase ya que estaba en la primera parte de su aula virtual, a comparación de los que no usaron aula virtual la cual indicaron que no sabían que tenían que lograr.

Los estudiantes que usaron el aula virtual estuvieron más motivados ya que comentaron que vieron un video que se encontraba en el aula virtual de un caso cotidiano relacionado con su carrera y que querían ser como los del video, además con el video pudieron conectar algunos conocimientos anteriores y relacionarlos con el nuevo tema, a comparación de los estudiantes que no usaron aula virtual la cual no mostraron ese interés en el nuevo tema. Los estudiantes que usaron el aula virtual mencionaron que les sirvió los foros, los enlaces, las retroalimentaciones en línea, como también los materiales didácticos e interactivos, a comparación de los estudiantes que no usaron el aula virtual y solo se quedaron con lo explicado en clase la cual manifestaron al final que tenían dudas. Al final en la evaluación de conocimientos sobre el tema trabajado el grupo de estudiantes que uso el aula virtual salió con mejores notas que el otro grupo que no uso aula virtual.

Como conclusión final, los datos de este estudio nos permiten observar que el uso del aula virtual mejora significativamente los procesos pedagógicos que se dan en una sesión de clase la cual nos llevaría al éxito de los aprendizajes.

\section{REFERENCIAS}

Aguilar, M. (2014). Influencia de las aulas virtuales en el aprendizaje por competencias de los estudiantes del curso de internado estomatológico [tesis doctoral, Universidad de San Martín de Porres]. http://www. repositorioacademico.usmp.edu.pe/bitstream/handle/usmp/1069/aguilar_ vm.pdf;jsessionid=751ABD9DE4C9AA9C301B495B8855EFA6? sequence=1 
Cepeda, L. G. (2017). Estrategia metodológica del uso de aulas virtuales en el proceso de enseñanza aprendizaje universitario. [tesis docotral, Universidad Nacional Mayor de San Marcos]. http://cybertesis.unmsm.edu.pe/bitstream/handle/20.500.12672/6058/ Cepeda_al.pdf? sequence $=1$

García, S. (2015). Análisis del uso de las aulas virtuales como apoyo a la formación presencial en el programa ingeniería de sonido [tesis de pregrado, Universidad de San Buenaventura]. http://biblioteca.usbbog.edu.co:8080/Biblioteca/BDigital/137674. pdf

Las Rutas del Aprendizaje. (08 de Junio de 2017). Procesos didácticos y pedagógicos CN 2017. Universidad Peruana Cayetano Heredia. https://lasrutasdelaprendizaje. blogspot.com/p/procesos-pedagogicos-en-la-sesion-de.html

Minedu. (2014). Marco de Buen Desempeño Docente. Corporación Gráfica Navarrete.

Rodrigo, D. (2015). La Educación Virtual Universitaria como medio para mejorar las competencias genéricas y los aprendizajes a través de buenas prácticas docentes [tesis doctoral, Universitat Politècnica de Catalunya]. https://www.tdx.cat/bitstream/ handle/10803/397710/TRADR1de1.pdf? sequence=1\&isAllowed=y

Web del maestro CMF. (2015). Los Procesos pedagógicos en la Sesión de aprendizaje. https://webdelmaestrocmf.com/portal/los-procesos-pedagogicos-en-la-sesion-deaprendizaje-2/ 